\title{
The utility of troponin measurement to detect myocardial infarction: review of the current findings
}

This article was published in the following Dove Press journal:

Vascular Health and Risk Management

30 July 2010

Number of times this article has been viewed

\author{
Melissa A Daubert \\ Allen Jeremias \\ Division of Cardiovascular Medicine, \\ Department of Internal Medicine, \\ Stony Brook University Medical \\ Center, Stony Brook, NY, USA
}

\begin{abstract}
Myocardial infarction (MI) is defined by the presence of myocardial necrosis in combination with clinical evidence of myocardial ischemia. Cardiac troponins are regulatory proteins within the myocardium that are released into the circulation when damage to the myocyte has occurred. Therefore, serum troponin is an exquisitely sensitive marker of myocardial injury and is necessary for establishing the diagnosis of MI. High-sensitivity troponin assays are improving the diagnostic accuracy and rapid detection of myocardial infarction. The early identification of MI is vital for the institution of anti-thrombotic therapy to limit myocardial damage and preserve cardiac function. Troponin has both diagnostic and prognostic significance in the setting of acute coronary syndrome (ACS). Increased troponin levels in the absence of ACS should prompt an evaluation for an alternative, non-thrombotic mechanism of troponin elevation and direct management at the underlying cause. This review describes the role of troponin in the evaluation of patients with suspected myocardial infarction.
\end{abstract}

Keywords: myocardial infarction, troponin, high-sensitivity assays

\section{Introduction}

Myocardial infarction (MI) describes the process of myocardial cell death due to ischemia, or the perfusion imbalance between supply and demand within the coronary arteries as a result of an acute thrombotic process. In 2006, approximately 16.8 million (7.6\%) people had a diagnosis of coronary heart disease in the United States. ${ }^{1}$ In the same year, an estimated 935,000 people experienced an acute MI, of which more than 150,000 resulted in death. ${ }^{1}$ Therefore, the early detection and diagnosis of MI is vital for the institution of therapy to limit myocardial damage and preserve cardiac function.

Acute coronary syndrome (ACS) refers to the constellation of clinical symptoms caused by active myocardial ischemia. The pathology underlying the development of ACS results from the erosion and rupture of a fibrous cap containing a lipid-rich atherosclerotic plaque that precipitates thrombus formation within the coronary artery. ${ }^{2}$ This pathologic process can result in a continuum of presentations among patients experiencing ACS. Patients exhibiting clinical symptoms of ischemia but with no evidence of myocardial necrosis based on serum biomarkers are considered to have unstable angina, ${ }^{3}$ whereas those patients who have positive cardiac biomarkers and demonstrate ischemic symptoms, with or without electrocardiographic ST-segment depression or T wave inversion, are experiencing non-ST elevation myocardial infarction (NSTEMI). Further along the ACS spectrum are patients with new ST-segment elevation on the electrocardiogram (ECG), which is diagnostic of acute ST-elevation myocardial infarction (STEMI). ${ }^{3}$ Clinical trials have clearly established the benefit of early reperfusion
Correspondence: Allen Jeremias

Stony Brook University Medical Center, Health Sciences Center, TI6-080,

Stony Brook, NY I I794, USA

Tel + | 63| $444 \mid 064$

Fax + I 63I 444 I054

Email allen.jeremias@stonybrook.edu 
therapy in $\mathrm{STEMI}^{4,5}$ and an early invasive strategy in patients with NSTEMI. ${ }^{6}$ Therefore, a rapid and accurate assessment of patients with ACS is essential for optimal management. ${ }^{3,7}$ This review describes the role of troponin in the evaluation of patients with suspected myocardial infarction.

\section{Historical evolution of defining myocardial infarction}

Considerable advances in the detection of myocardial injury and necrosis have been made in the last several decades. As a result, the definition of MI has evolved over time. Beginning in the 1950s, the World Health Organization used epidemiologic data to define MI as the presence of at least two of the following three criteria: 1 . clinical symptoms suggestive of myocardial ischemia, 2. ECG abnormalities, and 3. elevation in serum biomarkers indicative of myocardial necrosis. ${ }^{8}$ By 2000, the European Society of Cardiology (ESC) and the American College of Cardiology (ACC) established troponin as the biomarker of choice in the diagnosis of myocardial infarction. ${ }^{9}$ The development of increasingly sensitive and specific assays for the detection of myocardial necrosis, as well as the emergence of more precise imaging techniques for ischemic myocardial dysfunction, led to further refinement of the definition of MI. In 2007, a Global Task Force assembled from the ESC, ACC, American Heart Association, and World Heart Federation published a consensus statement that sought to standardize cardiac troponin detection, incorporate cardiac imaging, and classify myocardial infarctions based on etiology, thus furthering the evolution of the definition of MI. ${ }^{10}$

\section{Definition of myocardial infarction}

Acute MI is defined by the presence of myocardial necrosis in combination with the clinical presentation of myocardial ischemia. The diagnosis of acute myocardial infarction requires the rise and/or fall of cardiac biomarkers (preferably troponin) with at least one value above the 99th percentile of the upper reference limit (URL) in a healthy population. In addition, at least one of the following must be present: symptoms of ischemia; ECG changes indicative of active ischemia (new ST-T wave changes, new left bundle branch block, or the development of new pathologic Q waves) and/or imaging evidence of new regional wall motion abnormality; or the loss of viable myocardium. ${ }^{10}$

The rise and/or fall in serial troponin measurements are essential for the diagnosis of MI, and may also be necessary to distinguish acute MI from baseline elevated troponin levels. ${ }^{11}$ Detection of a dynamic troponin pattern demonstrates the acuity of myocardial injury and assists in narrowing the differential diagnosis. Experts have suggested that the degree of troponin change $(>20 \%)$ is another important characteristic that significantly improves specificity and may help to differentiate MI from other etiologies of elevated troponins, thereby avoiding diagnostic misclassification. ${ }^{12-14}$

Prior MI is distinguished from acute MI by the presence of pathological Q waves on ECG, or imaging evidence of myocardial loss (ie, a region that is thinned and fails to contract) in the absence of ischemia. ${ }^{10}$

\section{Types of myocardial infarction}

Myocardial infarctions are classified by the etiology of the ischemia (Table 1). ${ }^{10}$ Type 1 MIs are due to a primary coronary event such as the spontaneous rupture of an atherosclerotic plaque or dissection within the coronary artery resulting in STEMI or NSTEMI. Type 2 MIs are the result of a non-thrombotic condition causing an imbalance between coronary oxygen supply and demand leading to myocardial ischemia. Anemia, arrhythmias, hypertension, coronary artery spasm, and hypotension in the presence of fixed coronary disease are all possible precipitants of type 2 MIs. Sudden cardiac death defines the third type of MI. The

Table I Classification of myocardial infarction

\begin{tabular}{|c|c|}
\hline Type I & $\begin{array}{l}\text { Spontaneous myocardial infarction as the } \\
\text { result of a primary coronary event, such } \\
\text { as coronary artery plaque erosion and/or } \\
\text { rupture, fissure, or dissection. }\end{array}$ \\
\hline Type 2 & $\begin{array}{l}\text { Myocardial infarction associated with } \\
\text { ischemia secondary to either increased } \\
\text { oxygen demand or decreased supply, } \\
\text { such as in coronary artery spasm, } \\
\text { coronary embolism, anemia, arrhythmia, } \\
\text { hypertension, or hypotension. }\end{array}$ \\
\hline Type 3 & $\begin{array}{l}\text { Sudden unexpected cardiac death, including } \\
\text { cardiac arrest, often with symptoms } \\
\text { suggestive of myocardial ischemia, } \\
\text { accompanied by new ST-elevation, new } \\
\text { left bundle branch block, or evidence of } \\
\text { fresh thrombus in a coronary artery by } \\
\text { angiography and/or at autopsy, but death } \\
\text { occurring before blood samples could } \\
\text { be obtained, or at a time before the } \\
\text { appearance of cardiac biomarkers in the } \\
\text { blood. }\end{array}$ \\
\hline Type 4a & $\begin{array}{l}\text { Myocardial infarction associated with } \\
\text { percutaneous coronary intervention. }\end{array}$ \\
\hline Type 4b & $\begin{array}{l}\text { Myocardial infarction associated with stent } \\
\text { thrombosis as documented by angiography } \\
\text { or autopsy. }\end{array}$ \\
\hline Type 5 & $\begin{array}{l}\text { Myocardial infarction associated with } \\
\text { coronary artery bypass grafting. }\end{array}$ \\
\hline
\end{tabular}

Note: Modified and reproduced with permission from Thygesen et al. ${ }^{10}$ 
fourth classification is composed of two subtypes: a) percutaneous coronary intervention- (PCI) associated MI, which is defined as a biomarker increase that exceeds 3 times the 99th percentile of the URL; b) MI due to stent thrombosis. Type 5 MIs are secondary to coronary artery bypass grafting $(\mathrm{CABG})$ and by convention are defined as a biomarker increase that exceeds 5 times the 99th percentile of the URL, in combination with electrocardiographic, imaging, or angiographic evidence of ischemia.

\section{Biomarkers of myocardial infarction}

Cardiac biomarkers are an essential component of the criteria used to establish the diagnosis of acute myocardial infarction. The ideal biochemical marker should be in high concentration in the myocardium, absent in non-cardiac tissue, released rapidly in a linear fashion following myocardial necrosis, and present in the circulation long enough to be easily detectable by a relatively inexpensive and widely available assay.

The earliest biomarkers employed in the detection of ischemia included aspartate aminotransferase, total lactate dehydrogenase, and lactate dehydrogenase isoenzymes. ${ }^{15}$ However, these biomarkers have a wide tissue distribution that significantly limits the specificity for myocardial necrosis, and therefore these biomarkers should no longer be employed in the evaluation of acute MI. The next generation of cardiac biomarkers included creatine kinase (CK), which is a cytosolic carrier protein for high-energy phosphates. ${ }^{16}$ Creatine kinase MB (CK-MB) is an isoenzyme of creatine kinase that is most abundant in the heart. However, CK-MB also constitutes $1 \%-3 \%$ of the creatine kinase in skeletal muscle and is present in a small fraction in other organs such as the small bowel, uterus, prostate, and diaphragm. ${ }^{17}$ Therefore, the specificity of CK-MB can be reduced in the setting of major injury to these organs, especially skeletal muscle. When compared to CK-MB and other cardiac biomarkers, troponin (I or T) has demonstrated nearly absolute myocardial tissue specificity as well as high clinical sensitivity for myocardial ischemia. ${ }^{18,19}$ Thus, with the development and clinical availability of troponin assays, troponin has largely supplanted CK-MB for the initial detection of MI. Troponin is the preferred biomarker for the detection of myocardial necrosis and is a Class I indication for the diagnosis of MI. ${ }^{10,19,20}$

\section{Cardiac troponins}

Cardiac troponins are regulatory proteins that control the calcium-mediated interaction of actin and myosin, which results in contraction and relaxation of striated muscle. The troponin complex is made up of three subunits: troponin C, which binds calcium; troponin I, which inhibits actin-myosin interactions; and troponin $\mathrm{T}$, which attaches the troponin complex by binding to tropomysin, and facilitates contraction. Troponin $\mathrm{C}$ is expressed by cells in both cardiac and skeletal muscle. In contrast, the amino acid sequences of troponins I and T are unique to cardiac muscle. This difference has allowed for the development of rapid, quantitative assays to detect elevations of cardiac troponins in the serum. The plasma troponin level in healthy subjects is hypothesized to be $0.1-0.2 \mathrm{ng} / \mathrm{L}$, due to the continuous microscopic loss of cardiomyocytes during normal life. ${ }^{21}$

The majority of troponin is structurally bound in the contractile apparatus of the myofibril, but approximately $7 \%$ of troponin T and 3\%-5\% of troponin I is free in the cytoplasm. ${ }^{22}$ After damage to the myocyte occurs, there is a biphasic rise in serum troponin that corresponds to the initial release of free cytoplasmic troponin, followed by the gradual dispersion of myofibril-bound troponin complexes. ${ }^{23,24}$ Transmural necrosis of the myocardium requires at least 2-4 hours and may be even longer in the cases of pre-conditioning, collateral circulation, or intermittent coronary artery occlusion. ${ }^{10}$ Although troponin kinetics do not reliably permit the very early detection (initial 1-2 hours) of myocardial necrosis, troponin can be detected approximately 2-4 hours after the onset of myocardial injury. ${ }^{16,19}$ Therefore, blood samples are recommended to be drawn both at presentation and 6-9 hours later to optimize both the clinical sensitivity for ruling in MI and the specificity for ruling out MI. ${ }^{10,19}$ Serum levels can remain elevated for up to 4-7 days for troponin I, and 10-14 days for troponin T. ${ }^{25}$ Although the exact mechanism of troponin elimination is unknown, given its relatively large molecular size, troponin is believed to be cleared by the reticuloendothelial system. ${ }^{26}$ However, recent evidence suggests that troponin $\mathrm{T}$ is fragmented into molecules small enough to be renally excreted, which may explain the high prevalence of troponin $\mathrm{T}$ elevation in patients with renal failure. $^{27}$

\section{Troponin sensitivity and specificity}

Troponin kinetics dictate that the sensitivity of troponin improves with time. Using conventional assays, the sensitivity of troponin $\mathrm{T}$ at the time of hospital admission ranges from $25 \%-65 \%$, and increases to $59 \%-90 \%$ at 2 to 6 hours after presentation. ${ }^{28-30}$ The sensitivity approaches $100 \%$ by 6 to 12 hours after admission. ${ }^{28,29}$ The sensitivity of troponin I upon admission is less than $45 \%$, which improves to $69 \%-82 \%$ when measured 2 to 6 hours later and, similar 
to troponin T, achieves $100 \%$ sensitivity between 6 and 12 hours after admission. ${ }^{28-30}$ Thus, the maximal sensitivity of standard troponin assays is not achieved until 6 or more hours after the initiation of myocardial necrosis. ${ }^{16,31}$ Therefore, blood samples for the measurement of troponin levels are recommended to be drawn both at presentation and 6-9 hours later to optimize both the clinical sensitivity and specificity for the diagnosis of MI. ${ }^{19}$ The positive predictive value of troponin also increases with serial testing, improving from $25 \%$ for troponin I and 35\% for troponin $\mathrm{T}$ at presentation to $89 \%$ for troponin I and $57 \%$ for troponin $\mathrm{T}$ after 12 hours. $^{29}$

Specificity does not vary significantly over time. The specificity of troponin I is on the order of 83 to 98 percent with serial testing. ${ }^{29,30,32}$ Troponin $\mathrm{T}$ has specificities ranging from $86 \%-98 \% .^{29,30}$ The negative predictive value of troponin I and $\mathrm{T}$ at presentation is $85 \%$ and $88 \%$ respectively, and increases to $98 \%$ and $99 \%$ respectively after 12 hours. ${ }^{29}$ As a result of high tissue specificity, cardiac troponin is associated with fewer false-positive results in the setting of concomitant skeletal muscle injury than other biomarkers such as CK-MB. This inherent characteristic of troponin has been utilized in the assessment of myocardial injury in patients with chronic muscle diseases, crush injuries, marathon runners, following electrical cardioversion, and in the setting of perioperative myocardial infarctions. ${ }^{32-35}$ It should be noted that the tissue specificity of cardiac troponin is distinct from the specificity for the mechanism of myocardial injury such that, if elevated troponins are found in the absence of myocardial ischemia, an evaluation for alternative etiologies of myocardial injury should be pursued.

\section{Troponin assays}

In an effort to standardize the diagnosis of myocardial infarction and troponin measurements, the 2007 consensus definition required a concentration of cardiac troponin exceeding the 99th percentile of the upper reference limit in a healthy population on at least 1 occasion in the setting of clinical ischemia. ${ }^{10}$ Until recently however, there was no clinically available assay capable of consistently achieving this recommended precision. With the advent of the highlysensitive (hs) troponin assay, it is now possible to accurately measure troponin concentrations with the currently recommended level of precision. ${ }^{36}$ These new generation assays can measure troponin concentrations approximately 10-fold lower than conventional assays, and as a result, the 99th percentile concentration continues to decrease. For example, the 99th percentile value for the first-generation troponin $\mathrm{T}$ assay was
$0.06 \mathrm{ug} / \mathrm{L}$, which was reduced to $<0.01 \mathrm{ug} / \mathrm{L}$ by the fourthgeneration assays. ${ }^{37}$

\section{High-sensitivity (hs) troponin assays}

Troponin kinetics can complicate the very early detection of MI. However, newer generations of highly-sensitive troponin assays are helping to overcome this limitation. High-sensitivity assays for both troponin T and I are commercially available and are beginning to come into clinical use. Advances in immunoassay technology have resulted in multiple second-generation troponin I assays and a fourthgeneration troponin $\mathrm{T}$ assay, with a fifth-generation assay in development. ${ }^{37}$ The troponin $\mathrm{T}$ assays are produced by a single manufacturer, making results comparable. In contrast, there are several methodologies employed in troponin I assays across many manufacturers, and a lack of calibrator standardization has resulted in significant variation in troponin I results among different assays. ${ }^{37}$

Apple et al studied a second-generation hs-troponin I assay to determine the diagnostic accuracy for acute MI. The clinical sensitivity and specificity of the presenting blood sample from patients with ischemic symptoms suggestive of ACS was $69 \%$ and $78 \%$ respectively, which improved to $94 \%$ and $81 \%$ respectively 6 hours after presentation. ${ }^{14}$ Another study, using a different hs-troponin I assay, found the clinical sensitivity to be $90.7 \%$ at presentation with a specificity of $90.2 \%$ and a positive predictive value of $87 \%$, which demonstrates a much higher diagnostic accuracy than that of conventional troponin assays. ${ }^{38}$ In particular, among patients who presented within 3 hours of symptom onset, it was shown that a single hs-troponin I value above the 99th percentile value accurately predicted which patients would go on to have a $30 \%$ rise in the troponin I level within 6 hours. This suggests that one hs-troponin I level upon presentation was as effective at diagnosing MI as detecting serially rising troponin levels over the initial 6 hours. ${ }^{38}$ Conversely, the high specificity of hs-troponin assays, with negative predictive values of $97 \%-99 \%$, helped to reliably rule out $\mathrm{MI}$ on the basis of an initial measurement. ${ }^{39}$ Reichlin and colleagues also demonstrated the superior diagnostic precision of multiple high-sensitivity troponin I and $\mathrm{T}$ assays for the detection of acute MI as compared to a standard conventional troponin assay, especially among patients who presented within 3 hours of symptom onset. ${ }^{39}$ In addition, the diagnostic performance for NSTEMI, STEMI, and among patients with renal insufficiency was similar, regardless of which hs-troponin assay (ie, I or T) was used. ${ }^{39}$ 
The greater diagnostic accuracy of the hs-troponin assays allows for the opportunity to rapidly initiate effective medical management, including identifying patients who are candidates for early invasive procedures. ${ }^{39,40}$ However, future studies are still needed to determine if clinical outcomes are improved when patients are managed based on the highly-sensitive troponin results, especially the subgroup who now have positive hs-troponin values but would have had a negative result with a conventional (less sensitive) assay.

The improvement in the analytical sensitivities of troponin assays is likely to result in a diagnostic shift from a reduction in the diagnosis of unstable angina to a corresponding increased frequency of NSTEMI. ${ }^{41}$ In addition to this diagnostic shift, it is anticipated that a diagnostic dilemma will arise from the controversy over whether increasingly sensitive assays will result in the overdiagnosis of MI because pathologic mechanisms other than ischemia are being detected. ${ }^{13}$ The positive results of such patients in the hs-troponin trials may have inflated the diagnostic accuracy of these assays. ${ }^{42}$ This underscores the importance of using troponin levels and the temporal pattern in conjunction with a detailed clinical assessment.

Multiple studies of hs-troponin assays have demonstrated a high level of accuracy for the early diagnosis of MI. However, even when hs-troponin assays become widely available and cutoffs at the 99th percentile are consistently employed, it is still imperative to consider the clinical scenario, ECG findings, and potentially, adjunctive imaging techniques for the rapid and accurate diagnosis of MI.

\section{Troponin: risk stratification and management}

Cardiac troponin is a class I indication for risk stratification in patients with ACS. ${ }^{19}$ Several studies have demonstrated that in patients with ACS, increased concentrations of troponin closely correlate with the presence, complexity, and severity of epicardial coronary artery disease, as well as decreased microvascular myocardial perfusion. ${ }^{43,44}$

Troponin results can help to guide patient management. Morrow et al demonstrated that patients with NSTEMI had a large clinical benefit (approximately 55\% reduction in the odds of death or MI) when an early invasive strategy was employed versus conservative management, even in patients with only minor troponin elevations. ${ }^{45}$ Conversely, early angiography and revascularization was not associated with a detectable benefit in patients who did not have an increased concentration of troponin. ${ }^{45}$ In the case of STEMI however, reperfusion therapy should not be delayed waiting for confirmatory biomarkers of myocardial injury.

The temporal pattern and peak troponin value can also be clinically useful in MI management. The temporal pattern, or degree of change in troponin values, allows the clinician to distinguish between acute MI and chronic troponin elevations. ${ }^{13}$ The troponin trend can aid in the assessment of the success of reperfusion in an infarct-related artery following thrombolytic therapy. A rapid washout or an early peak in troponin levels has a positive predictive value of greater than $90 \%$ for infarct artery patency ${ }^{46,47}$ In patients for whom reinfarction is suspected based on clinical symptoms, immediate troponin measurement is recommended. ${ }^{10}$ Recurrent infarction is diagnosed if there is greater than a $20 \%$ increase in the troponin value. ${ }^{48,49}$

\section{Troponin: prognostic implications}

Cardiac troponins have not only diagnostic value, but yield prognostic information as well. Patients presenting with clinical evidence of ischemia and increased troponins have worse outcomes than those without detectable troponin in the circulation. ${ }^{38,50,51}$ Even in patients with stable coronary artery disease, high-sensitivity assays have demonstrated that detectable concentrations of cardiac troponin portend a higher incidence of heart failure and cardiovascular death. ${ }^{52}$ Prognosis is related in part to the extent of the increase in troponin in patients with an ischemic mechanism for myocardial injury. ${ }^{45,53,54}$ Recently, the MISSION! trial showed that peak troponin $\mathrm{T}$ levels are a good estimate of infarct size, and an independent predictor for left ventricular function at 3 months, and major adverse cardiac events at 1 year. ${ }^{54}$

Cardiac troponin has also been proven to be a potent, independent indicator of recurrent ischemic events, and an estimate for the risk of death among patients presenting with ACS..$^{55}$ The mortality risk appears to correlate with the level of troponin rise. The TIMI-IIIB trial demonstrated that in patients presenting with ACS, mortality was consistently higher among patients with elevated troponin I at the time of admission. There were statistically significant increases in mortality with increasing levels of troponin I, with a relative risk for death of 7.8 among the group with the highest level of troponin, even after adjustment for age $>65$, ST depression on ECG, and other baseline variables.$^{56}$ Additionally, the GUSTO IIa trial found that elevated troponin $\mathrm{T}$ was significantly predictive of 30-day mortality in patients with acute myocardial ischemia, even after analysis was adjusted for electrocardiographic category and CK-MB level. ${ }^{57}$ 


\section{Alternative, non-thrombotic mechanisms of troponin elevation}

Serum biomarkers of myocardial necrosis have a vital role in the detection of cardiac ischemia, but the diagnosis of MI is not predicated exclusively on the presence of increased biomarkers. The diagnosis of myocardial infarction should be used when both biomarkers are detected and the clinical setting is consistent with myocardial ischemia. Many disease states can be associated with an increase in cardiac biomarkers in the absence of ACS. These elevations arise from pathologic mechanisms other than thrombotic coronary artery occlusion, and require treatment of the underlying cause rather than the administration of antithrombotic and antiplatelet agents. ${ }^{16,58}$

Alternative, non-thrombotic causes and mechanisms of troponin elevation include tachycardia, heart failure, infiltrative disorders, myocarditis, sepsis, anemia, pulmonary embolus, intracranial hemorrhage, stroke, drug toxicity, and renal failure (Table 2). In addition, falsepositive troponin elevations can occur due to hemolysis and assay interference with heterophilic antibodies. ${ }^{37}$ It is estimated that heterophilic antibodies cause about one false result in every 2000 investigations with modern immunoassays. ${ }^{59}$ To minimize the occurrence of falsepositive troponins, non-specific blocking antibodies have been added to modern assays to reduce interference with the results. ${ }^{59}$

Table 2 Non-thrombotic causes of elevated troponin

Demand ischemia (in the absence of ACS)
Supraventricular tachycardia/atrial fibrillation
Left ventricular hypertrophy
Anemia
Hypotension
Hypovolemia
Direct myocardial damage
Cardiac contusion
Direct current cardioversion
Cardiac infiltrative disorders
Chemotherapy
Myocarditis
Cardiac transplantation (immune-mediated reactions)
Myocardial strain
Congestive heart failure
Pulmonary embolism
Pulmonary hypertension or COPD
Chronic renal insufficiency
Sepsis/systemic inflammatory processes
Intracranial pathology
Intracerebral hemorrhage or stroke
Abbreviations: ACS, acute coronary syndrome; COPD, chronic obstructive
pulmonary disease.

There are many cardiovascular states that result in increased troponin levels in the absence of overt ischemic heart disease, including supraventricular tachycardia, atrial fibrillation, cardiac amyloidosis, left ventricular hypertrophy, heart failure, cardiac contusion, myocarditis, and heart transplant rejection. Tachycardia can augment myocardial oxygen demand while decreasing myocardial oxygen supply, predominantly by reducing the time in diastole and thereby limiting myocardial perfusion. ${ }^{58}$ This can occur even in the absence of flow-limiting epicardial coronary stenosis. Elevated cardiac troponin has also been observed in the setting of left ventricular hypertrophy. The increased left ventricular mass necessitates a greater myocardial oxygen demand and may induce occult subendocardial ischemia. Hamwi and colleagues reported that among patients without any clinical evidence of active ischemia, patients in the upper tertile of left ventricular mass had increased troponin levels in comparison to those patients in the lowest tertile. ${ }^{60}$ Heart failure can lead to troponin release via both myocardial strain and myocyte death independent of myocardial ischemia. Myocardial strain is produced by biventricular volume and pressure overload, causing excessive wall tension with resultant myofibrillary damage. ${ }^{61}$ Direct myocardial damage can also predispose to increased troponin levels from cell injury due to trauma or local inflammation. Blunt trauma, as in cardiac contusion or cardiopulmonary resuscitation, as well as trauma due to ablation, cardioversion/defibrillation, and endomyocardial biopsy can result in troponin elevations. ${ }^{49,62}$ Focal inflammatory disorders including myocarditis and immune-mediated reactions after heart transplantation have also been associated with a rise in troponin. The level of troponin I elevation has been shown to directly correlate with the degree of myocardial inflammation. ${ }^{63}$

Troponin elevation is a common finding among critically ill patients and is associated with a significantly increased mortality. ${ }^{64}$ A study evaluating ICU patients, in whom coronary artery disease had been definitely excluded, found that the risk of death was fourfold higher in the group with increased troponins than in those patients without detectable elevations. ${ }^{65}$ Systemic inflammatory processes, including sepsis, can result in increased oxygen consumption, decreased perfusion pressure, extrinsic myocardial depression, and subsequent troponin release.$^{66}$ No definitive causal relationship has been demonstrated, but it has been proposed that the inflammatory mediators such as $\mathrm{C}$-reactive protein, tumor necrosis factor-alpha, and interleukin-6 may contribute to the myocardial oxygen demand-supply 
mismatch. ${ }^{65}$ In addition, it has been proposed that myocardial depressive factors (released in the setting of sepsis and other inflammatory states) cause degradation of free troponin, in situ, to lower molecular weight fragments. ${ }^{67}$ With increased membrane permeability, those smaller troponin fragments could be released into the systemic circulation. In this setting, myocyte damage may not be permanent, and thus cell necrosis does not occur. This notion is supported by the clinical observation that myocardial depression during sepsis is a fully reversible process in most surviving patients. ${ }^{68}$ Increased troponin levels have also been observed in pulmonary embolism, pulmonary hypertension, and chronic obstructive pulmonary disease, presumably secondary to right heart strain. The reported incidence of troponin elevation among patients with acute pulmonary embolism varies from $16 \%$ to $50 \%$, and elevated levels are associated with a significant increase in mortality. ${ }^{69-71}$

Patients with intracranial pathology such as hemorrhage or acute stroke are frequently found to have elevated troponin levels along with ischemic changes on the ECG. As many as $20 \%$ of patients with subarachnoid hemorrhages, and up to $27 \%$ with acute stroke symptoms, have increased levels of troponin. ${ }^{72,73}$ Substantial elevation of troponin I in the setting of subarachnoid hemorrhage has been shown to indicate on ominous prognosis. It has been proposed that a deregulation of the autonomic nervous system in response to these intracranial processes results in excessive sympathetic activity and, ultimately, an increased catecholamine effect on the myocardium. ${ }^{74}$

Persistently elevated cardiac troponin is frequently observed among patients with end-stage renal disease (ESRD). ${ }^{26,75,76}$ The prevalence of increased troponin among asymptomatic ESRD patients may be as high as $53 \%{ }^{26}$ This may be the result of small areas of clinically silent myocardial necrosis, but other causes, such as increased left ventricular mass and impaired troponin excretion, have also been proposed. . $^{27,77-79}$

The presence of troponin elevation in a multitude of nonthrombotic disease states has been associated with increased short- and long-term mortality. The reasons for this impaired survival are unclear. However, cardiac troponin release may be indicative of more severe or extensive disease. Therefore, patients with elevated cardiac troponin levels should be evaluated for ACS. If this is excluded, then a thorough diagnostic evaluation for the non-thrombotic etiology of the troponin rise should be performed, and subsequent management should be directed at treating the underlying disorder. Patients with a non-thrombotic condition are not likely to derive benefit from the antithrombotic and/or invasive revascularization therapies that are commonly utilized in ACS.

\section{Conclusion}

The early detection of MI is crucial to the preservation of cardiac function. Troponin is an exquisitely sensitive marker of myocardial necrosis, and is necessary for establishing the diagnosis of $\mathrm{MI}$ in a clinical setting consistent with ischemia. Myocardial infarctions are classified by the etiology of the ischemia, which influences subsequent management strategies. Further studies are needed to determine if the greater diagnostic accuracy of high-sensitivity troponin assays will improve the clinical outcomes in patients experiencing $\mathrm{ACS}$. In the setting of ACS, troponins have not only diagnostic, but prognostic importance as well. Elevated troponin levels in the absence of ACS should prompt an evaluation for a non-thrombotic mechanism of troponin increase.

\section{Disclosure}

The authors have no disclosures or conflicts of interest with respect to this work.

\section{References}

1. Lloyd-Jones D, Adams R, Carnethon M, et al. Heart disease and stroke statistics - 2009 update: a report from the American Heart Association Statistics Committee and Stroke Statistics Subcommittee. Circulation. 2009;119(3):480-486.

2. Hansson GK. Inflammation, atherosclerosis, and coronary artery disease. N Engl J Med. 2005;352(16):1685-1695.

3. Anderson JL, Adams CD, Antman EM, et al. ACC/AHA 2007 guidelines for the management of patients with unstable angina/non-ST-Elevation myocardial infarction: a report of the American College of Cardiology/ American Heart Association Task Force on Practice Guidelines (Writing Committee to Revise the 2002 Guidelines for the Management of Patients With Unstable Angina/Non-ST-Elevation Myocardial Infarction) developed in collaboration with the American College of Emergency Physicians, the Society for Cardiovascular Angiography and Interventions, and the Society of Thoracic Surgeons endorsed by the American Association of Cardiovascular and Pulmonary Rehabilitation and the Society for Academic Emergency Medicine. J Am Coll Cardiol. 2007; 50(7):e1-e157.

4. Weaver WD, Simes RJ, Betriu A, et al. Comparison of primary coronary angioplasty and intravenous thrombolytic therapy for acute myocardial infarction: a quantitative review. JAMA. 1997;278(23): 2093-2098.

5. Keeley EC, Boura JA, Grines CL. Primary angioplasty versus intravenous thrombolytic therapy for acute myocardial infarction: a quantitative review of 23 randomised trials. Lancet. 2003;361(9351):13-20.

6. Cannon CP, Weintraub WS, Demopoulos LA, et al. Comparison of early invasive and conservative strategies in patients with unstable coronary syndromes treated with the glycoprotein IIb/IIIa inhibitor tirofiban. N Engl J Med. 2001;344(25):1879-1887.

7. Antman EM, Hand M, Armstrong PW, et al. 2007 focused update of the ACC/AHA 2004 guidelines for the management of patients with ST-elevation myocardial infarction: a report of the American College of Cardiology/American Heart Association Task Force on Practice Guidelines. J Am Coll Cardiol. 2008;51(2):210-247. 
8. Luepker RV, Apple FS, Christenson RH, et al. Case definitions for acute coronary heart disease in epidemiology and clinical research studies: a statement from the AHA Council on Epidemiology and Prevention; AHA Statistics Committee; World Heart Federation Council on Epidemiology and Prevention; the European Society of Cardiology Working Group on Epidemiology and Prevention; Centers for Disease Control and Prevention; and the National Heart, Lung, and Blood Institute. Circulation. 2003;108(20):2543-2549.

9. Alpert JS, Thygesen K, Antman E, Bassand JP. Myocardial infarction redefined - a consensus document of The Joint European Society of Cardiology/American College of Cardiology Committee for the redefinition of myocardial infarction. $J$ Am Coll Cardiol. 2000;36(3):959-969.

10. Thygesen K, Alpert JS, White HD. Universal definition of myocardial infarction. J Am Coll Cardiol. 2007;50(22):2173-2195.

11. Jaffe AS. Chasing troponin: how low can you go if you can see the rise? J Am Coll Cardiol. 2006;48(9):1763-1764.

12. Wu AH, Jaffe AS. The clinical need for high-sensitivity cardiac troponin assays for acute coronary syndromes and the role for serial testing. Am Heart J. 2008;155(2):208-214.

13. Eggers KM, Lind L, Venge P, Lindahl B. Will the universal definition of myocardial infarction criteria result in an overdiagnosis of myocardial infarction? Am J Cardiol. 2009;103(5):588-591.

14. Apple FS, Pearce LA, Smith SW, Kaczmarek JM, Murakami MM. Role of monitoring changes in sensitive cardiac troponin I assay results for early diagnosis of myocardial infarction and prediction of risk of adverse events. Clin Chem. 2009;55(5):930-937.

15. Apple FS, Jesse RL, Newby LK, Wu AH, Christenson RH. National Academy of Clinical Biochemistry and IFCC Committee for Standardization of Markers of Cardiac Damage Laboratory Medicine Practice Guidelines: Analytical issues for biochemical markers of acute coronary syndromes. Circulation. 2007;115(13):e352-e355.

16. Jaffe AS, Babuin L, Apple FS. Biomarkers in acute cardiac disease: the present and the future. J Am Coll Cardiol. 2006;48(1):1-11.

17. Roberts R, Sobel BE. Editorial: Isoenzymes of creatine phosphokinase and diagnosis of myocardial infarction. Ann Intern Med. 1973; 79(5):741-743.

18. Gerhardt W, Nordin G, Ljungdahl L. Can troponin T replace CK MBmass as "gold standard" for acute myocardial infarction ("AMI")? Scand J Clin Lab Invest Suppl. 1999;230:83-89.

19. Morrow DA, Cannon CP, Jesse RL, et al. National Academy of Clinical Biochemistry Laboratory Medicine Practice Guidelines: clinical characteristics and utilization of biochemical markers in acute coronary syndromes. Clin Chem. 2007;53(4):552-574.

20. Jaffe AS, Ravkilde J, Roberts R, et al. It's time for a change to a troponin standard. Circulation. 2000;102(11):1216-1220.

21. Missov ED, De Marco T. Clinical insights on the use of highly sensitive cardiac troponin assays. Clin Chim Acta. 1999;284(2):175-185.

22. Wu AH, Feng YJ. Biochemical differences between cTnT and cTnI and their significance for diagnosis of acute coronary syndromes. Eur Heart J. 1998;19 Suppl N:N25-N29.

23. Higgins JP, Higgins JA. Elevation of cardiac troponin I indicates more than myocardial ischemia. Clin Invest Med. 2003;26(3):133-147.

24. Katus HA, Remppis A, Scheffold T, Diederich KW, Kuebler W. Intracellular compartmentation of cardiac troponin $\mathrm{T}$ and its release kinetics in patients with reperfused and nonreperfused myocardial infarction. Am J Cardiol. 1991;67(16):1360-1367.

25. Adams JE 3rd, Abendschein DR, Jaffe AS. Biochemical markers of myocardial injury. Is MB creatine kinase the choice for the 1990s? Circulation. 1993;88(2):750-763.

26. Freda BJ, Tang WH, van Lente F, Peacock WF, Francis GS. Cardiac troponins in renal insufficiency: review and clinical implications. $\mathrm{J} \mathrm{Am}$ Coll Cardiol. 2002;40(12):2065-2071.

27. Diris JH, Hackeng CM, Kooman JP, Pinto YM, Hermens WT, van Dieijen-Visser MP. Impaired renal clearance explains elevated troponin T fragments in hemodialysis patients. Circulation. 2004;109(1): $23-25$.
28. Panteghini M, Pagani F, Bonetti G. The sensitivity of cardiac markers: an evidence-based approach. Clin Chem Lab Med. 1999;37(11-12): 1097-1106.

29. Tucker JF, Collins RA, Anderson AJ, Hauser J, Kalas J, Apple FS. Early diagnostic efficiency of cardiac troponin I and Troponin T for acute myocardial infarction. Acad Emerg Med. 1997;4(1): 13-21.

30. Balk EM, Ioannidis JP, Salem D, Chew PW, Lau J. Accuracy of biomarkers to diagnose acute cardiac ischemia in the emergency department: a meta-analysis. Ann Emerg Med. 2001;37(5): 478-494.

31. Eggers KM, Oldgren J, Nordenskjold A, Lindahl B. Diagnostic value of serial measurement of cardiac markers in patients with chest pain: limited value of adding myoglobin to troponin I for exclusion of myocardial infarction. Am Heart J. 2004;148(4):574-581.

32. Adams JE 3rd, Bodor GS, Davila-Roman VG, et al. Cardiac troponin I. A marker with high specificity for cardiac injury. Circulation. 1993;88(1):101-106

33. Georges JL, Spentchian M, Caubel C, et al. Time course of troponin I, myoglobulin, and cardiac enzyme release after electrical cardioversion. Am J Cardiol. 1996;78(7):825-826.

34. Adams JE 3rd, Davila-Roman VG, Bessey PQ, Blake DP, Ladenson $\mathrm{JH}$, Jaffe AS. Improved detection of cardiac contusion with cardiac troponin I. Am Heart J. 1996;131(2):308-312.

35. Adams JE 3rd, Sicard GA, Allen BT, et al. Diagnosis of perioperative myocardial infarction with measurement of cardiac troponin I. $N$ Engl J Med. 1994;330(10):670-674.

36. Mohammed AA, Januzzi JL Jr. Clinical applications of highly sensitive troponin assays. Cardiol Rev. 18(1):12-19.

37. Tate JR. Troponin revisited 2008: assay performance. Clin Chem Lab Med. 2008;46(11):1489-1500.

38. Keller T, Zeller T, Peetz D, et al. Sensitive troponin I assay in early diagnosis of acute myocardial infarction. N Engl J Med. 2009;361(9): 868-877.

39. Reichlin T, Hochholzer W, Bassetti S, et al. Early diagnosis of myocardial infarction with sensitive cardiac troponin assays. NEngl J Med. 2009;361(9):858-867.

40. Venge P, Lagerqvist B, Diderholm E, Lindahl B, Wallentin L. Clinical performance of three cardiac troponin assays in patients with unstable coronary artery disease (a FRISC II substudy). Am J Cardiol. 2002;89(9):1035-1041.

41. Wilson SR, Sabatine MS, Braunwald E, Sloan S, Murphy SA, Morrow DA. Detection of myocardial injury in patients with unstable angina using a novel nanoparticle cardiac troponin I assay: observations from the PROTECT-TIMI 30 Trial. Am Heart J. 2009;158(3):386-391.

42. Jaffe AS, Apple FS. High-sensitivity cardiac troponin: hype, help, and reality. Clin Chem. 56(3):342-344.

43. Hamm CW, Heeschen C, Goldmann B, et al. Benefit of abciximab in patients with refractory unstable angina in relation to serum troponin $\mathrm{T}$ levels. c7E3 Fab Antiplatelet Therapy in Unstable Refractory Angina (CAPTURE) Study Investigators. $N$ Engl J Med. 1999;340(21): 1623-1629.

44. Wong GC, Morrow DA, Murphy S, et al. Elevations in troponin T and I are associated with abnormal tissue level perfusion: a TACTICSTIMI 18 substudy. Treat Angina with Aggrastat and Determine Cost of Therapy with an Invasive or Conservative Strategy-Thrombolysis in Myocardial Infarction. Circulation. 2002;106(2):202-207.

45. Morrow DA, Cannon CP, Rifai N, et al. Ability of minor elevations of troponins I and $\mathrm{T}$ to predict benefit from an early invasive strategy in patients with unstable angina and non-ST elevation myocardial infarction: results from a randomized trial. JAMA. 2001;286(19): 2405-2412.

46. Tanasijevic MJ, Cannon CP, Antman EM, et al. Myoglobin, creatinekinase-MB and cardiac troponin-I 60-minute ratios predict infarctrelated artery patency after thrombolysis for acute myocardial infarction: results from the Thrombolysis in Myocardial Infarction study (TIMI) 10B. J Am Coll Cardiol. 1999;34(3):739-747. 
47. de Lemos JA, Morrow DA, Gibson CM, et al. Early noninvasive detection of failed epicardial reperfusion after fibrinolytic therapy. $\mathrm{Am}$ J Cardiol. 2001;88(4):353-358.

48. Apple FS, Murakami MM. Cardiac troponin and creatine kinase MB monitoring during in-hospital myocardial reinfarction. Clin Chem. 2005;51(2):460-463.

49. Jaffe AS. The clinical impact of the universal diagnosis of myocardial infarction. Clin Chem Lab Med. 2008;46(11):1485-1488.

50. Kontos MC, Shah R, Fritz LM, et al. Implication of different cardiac troponin I levels for clinical outcomes and prognosis of acute chest pain patients. J Am Coll Cardiol. 2004;43(6):958-965.

51. Eggers KM, Lagerqvist B, Venge P, Wallentin L, Lindahl B. Persistent cardiac troponin I elevation in stabilized patients after an episode of acute coronary syndrome predicts long-term mortality. Circulation. 2007;116(17):1907-1914.

52. Omland T, de Lemos JA, Sabatine MS, et al. A sensitive cardiac troponin T assay in stable coronary artery disease. N Engl J Med. 2009;361(26): 2538-2547.

53. Stubbs P, Collinson P, Moseley D, Greenwood T, Noble M. Prognostic significance of admission troponin $\mathrm{T}$ concentrations in patients with myocardial infarction. Circulation. 1996;94(6):1291-1297.

54. Hassan AK, Bergheanu SC, Hasan-Ali H, et al. Usefulness of peak troponin-T to predict infarct size and long-term outcome in patients with first acute myocardial infarction after primary percutaneous coronary intervention. Am J Cardiol. 2009;103(6):779-784.

55. Heidenreich PA, Alloggiamento T, Melsop K, McDonald KM, Go AS, Hlatky MA. The prognostic value of troponin in patients with non-ST elevation acute coronary syndromes: a meta-analysis. $\mathrm{J}$ Am Coll Cardiol. 2001;38(2):478-485.

56. Antman EM, Tanasijevic MJ, Thompson B, et al. Cardiac-specific troponin I levels to predict the risk of mortality in patients with acute coronary syndromes. $N$ Engl J Med. 1996;335(18): 1342-1349.

57. Ohman EM, Armstrong PW, Christenson RH, et al. Cardiac troponin T levels for risk stratification in acute myocardial ischemia. GUSTO IIA Investigators. N Engl J Med. 1996;335(18):1333-1341.

58. Jeremias A, Gibson CM. Narrative review: alternative causes for elevated cardiac troponin levels when acute coronary syndromes are excluded. Ann Intern Med. 2005;142(9):786-791.

59. Shayanfar N, Bestmann L, Schulthess G, Hersberger M. False-positive cardiac troponin $\mathrm{T}$ due to assay interference with heterophilic antibodies. Swiss Med Wkly. 2008;138(31-32):470.

60. Hamwi SM, Sharma AK, Weissman NJ, et al. Troponin-I elevation in patients with increased left ventricular mass. Am J Cardiol. 2003; 92(1):88-90.

61. Horwich TB, Patel J, MacLellan WR, Fonarow GC. Cardiac troponin I is associated with impaired hemodynamics, progressive left ventricular dysfunction, and increased mortality rates in advanced heart failure. Circulation. 2003;108(7):833-838.

62. Hasdemir C, Shah N, Rao AP, et al. Analysis of troponin I levels after spontaneous implantable cardioverter defibrillator shocks. J Cardiovasc Electrophysiol. 2002;13(2):144-150.
63. Smith SC, Ladenson JH, Mason JW, Jaffe AS. Elevations of cardiac troponin I associated with myocarditis: experimental and clinical correlates. Circulation. 1997;95(1):163-168.

64. Guest TM, Ramanathan AV, Tuteur PG, Schechtman KB, Ladenson JH, Jaffe AS. Myocardial injury in critically ill patients. A frequently unrecognized complication. JAMA. 1995;273(24):1945-1949.

65. Ammann P, Maggiorini M, Bertel O, et al. Troponin as a risk factor for mortality in critically ill patients without acute coronary syndromes. J Am Coll Cardiol. 2003;41(11):2004-2009.

66. Ammann P, Fehr T, Minder EI, Gunter C, Bertel O. Elevation of troponin I in sepsis and septic shock. Intensive Care Med. 2001;27(6): 965-969.

67. $\mathrm{Wu} \mathrm{AH}$. Increased troponin in patients with sepsis and septic shock: myocardial necrosis or reversible myocardial depression? Intensive Care Med. 2001;27(6):959-961.

68. Parrillo JE. Pathogenetic mechanisms of septic shock. N Engl J Med. 1993;328(20):1471-1477.

69. Yalamanchili K, Sukhija R, Aronow WS, Sinha N, Fleisher AG, Lehrman SG. Prevalence of increased cardiac troponin I levels in patients with and without acute pulmonary embolism and relation of increased cardiac troponin I levels with in-hospital mortality in patients with acute pulmonary embolism. Am J Cardiol. 2004;93(2):263-264.

70. Aksay E, Yanturali S, Kiyan S. Can elevated troponin I levels predict complicated clinical course and inhospital mortality in patients with acute pulmonary embolism? Am J Emerg Med. 2007;25(2):138-143.

71. Becattini C, Vedovati MC, Agnelli G. Prognostic value of troponins in acute pulmonary embolism: a meta-analysis. Circulation. 2007;116(4): 427-433.

72. Tung P, Kopelnik A, Banki N, et al. Predictors of neurocardiogenic injury after subarachnoid hemorrhage. Stroke. 2004;35(2):548-551.

73. Trooyen M, Indredavik B, Rossvoll O, Slordahl SA. [Myocardial injury in acute stroke assessed by troponin I]. Tidsskr Nor Laegeforen. 2001;121(4):421-425.

74. Homma S, Grahame-Clarke C. Editorial comment - myocardial damage in patients with subarachnoid hemorrhage. Stroke. 2004;35(2): 552-553.

75. deFilippi C, Wasserman S, Rosanio S, et al. Cardiac troponin T and $\mathrm{C}$-reactive protein for predicting prognosis, coronary atherosclerosis, and cardiomyopathy in patients undergoing long-term hemodialysis. JAMA. 2003;290(3):353-359.

76. Apple FS, Murakami MM, Pearce LA, Herzog CA. Predictive value of cardiac troponin I and T for subsequent death in end-stage renal disease. Circulation. 2002;106(23):2941-2945.

77. Ooi DS, Zimmerman D, Graham J, Wells GA. Cardiac troponin T predicts long-term outcomes in hemodialysis patients. Clin Chem. 2001; 47(3):412-417.

78. Antman EM, Grudzien C, Mitchell RN, Sacks DB. Detection of unsuspected myocardial necrosis by rapid bedside assay for cardiac troponin T. Am Heart J. 1997;133(5):596-598.

79. Sharma R, Gaze DC, Pellerin D, et al. Cardiac structural and functional abnormalities in end stage renal disease patients with elevated cardiac troponin T. Heart. 2006;92(6):804-809.
Vascular Health and Risk Management

\section{Publish your work in this journal}

Vascular Health and Risk Management is an international, peerreviewed journal of therapeutics and risk management, focusing on concise rapid reporting of clinical studies on the processes involved in the maintenance of vascular health; the monitoring, prevention and treatment of vascular disease and its sequelae; and the involvement of
Dovepress

metabolic disorders, particularly diabetes. This journal is indexed on PubMed Central and MedLine. The manuscript management system is completely online and includes a very quick and fair peer-review system, which is all easy to use. Visit http://www.dovepress.com/ testimonials.php to read real quotes from published authors. 